\title{
Rigid Formation Construction from Non-rigid Components
}

\author{
Yun Hou, Changbin Yu
}

\begin{abstract}
This paper discusses the construction of rigid formation from arbitrary non-rigid components in twodimensional space. Specifically, we focus on developing strategies for the construction sequences under the premise of building minimum number of links between the non-rigid components. Three operations, namely spindle splitting, rigid component shrink and edge floating, are proposed. The scenarios of acquiring a rigid formation from different kinds of non-rigid components are discussed respectively. It is proved that our strategy will guarantee the rigidity of the obtained formation with minimum number of inserted links, and will cover all the possible solutions during the construction process.
\end{abstract}

\section{INTRODUCTION}

Formation control has been a hotspot in the research of multi-agent systems, and many solutions have been proposed by different predecessors.A common strategy adopted by many formation control solutions is to extract topology from the sensing or communication networks among agents as well as designing appropriate control laws. By saying topology, we mean to omit all the detailed physical interconnections, transmitting speeds or signal types, and to concentrate only on the interconnection edges between agents. Especially in distance-based control, formation can be kept only when all the constraints of distance are maintained throughout. A formation is defined as rigid if the distance of each pair of agents remain constant all the time. If a formation can be kept by maintaining a least number of interconnection links, it is then mentioned as minimally rigid formation. Minimal rigidity can certainly reduce the cost of communication or observation thus is a basic requirement in practical applications.

Minimally rigid formations can be obtained by the wellknown Henneberg Sequence, and lots of relevant works have been done to preserve minimal rigidity under different situations. In [4], a reversed Henneberg Sequence was proposed to regain minimal rigidity if some links or agents

Y. Hou was with Shandong Provincial Key Laboratory of Computer Network, Shandong Computer Science Center, Jinan 250014, China, and also with the Australian National University and NICTA Ltd., Canberra ACT 0200, Australia (e-mail: Yun.hou@anu.edu.au). The work of Y. Hou was supported by NICTA, which is funded by the Australian Government as represented by the Department of Broadband, Communications and the Digital Economy and the Australian Research Council (ARC) through the ICT Centre of Excellence program. C. Yu was with the Research School of Engineering, Australian National University and NICTA Ltd., Canberra ACT 0200, Australia, and also with Shandong Computer Science Center (e-mail: brad.yu@anu.edu.au). The work of C. Yu was supported in part by the Australian Research Council through Discovery Project DP130103610, a Queen Elizabeth II Fellowship under Grant DP110100538 and Overseas Expert Program of Shandong Province, and a grant from Shandong Academy of Science Development Fund for Science and Technology, and the Pilot Project for Science and Technology in Shandong Academy of Science, the Open Research Project of the State Key Laboratory of Industrial Control Technology, Zhejiang University, China. are removed from a minimally rigid formation. Closing ranks problem was studied in [2] and [3] and different self-repair approaches were proposed to regain rigidity for non-rigid formations.

Besides the Hennerberg sequence, the construction of rigid formation from rigid components were well studied. The merging of rigid formations was discussed in both two and three dimensional space in [5], where minimum number of links were inserted to regain minimal rigidity. In [7], three principles were proposed in the scenario of rigid formation merging to cover all the possible solutions, such that any scenario of rigid formation merging can be processed. Three basic solutions were proposed in [6] to obtain a rigid formation from two rigid ones in two-dimensional space. However, how to construct a rigid formation from non-rigid components is not discovered yet.

One motivation is that under some cases the rigidity of a given formation can not be repaired by approaches such as minimal cover and closing rank. A trivial example is presented in Fig. 1, where the rigidity of one formation can not be restored unless is connected with a rigid formation $F$. This scenario motivates us to develop a construction strategy to obtain rigid formation from non-rigid components, which can be employed in applications like obtaining a larger rigid formation by connecting two or more non-rigid ones, which is capable to deal with tasks that requires more agents.

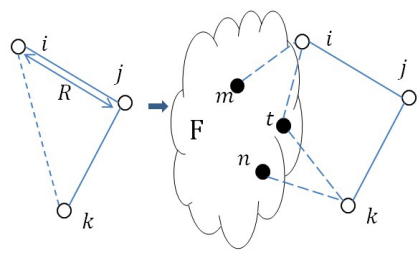

Fig. 1. An example showing that the rigidity of formation can not be restored by building new links within formation, because the distance of agent $i$ and $k$ exceeds the communication range $R$. In this case by connecting $i$ and $k$ to another formation, the rigidity will be restored.

The construction of rigid formation with non-rigid components is far more complicated comparing with the aforementioned three basic solutions proposed in [6]. In the nonrigid component case, some agents must be connected, while other agents can be left alone. Therefore the solutions are uniquely determined by the actual topologies of non-rigid components. One can of course argue that this is trivial since one can connect every two agents from the two nonrigid components to obtain a rigid one, but what makes the question nontrivial is how to pick up a minimum set of links in the process of construction. Note that all the inserted edges will be deployed only between the two non- 
rigid components, not within any of them. This problem may not seem to be feasible since a direct approach similar to rigid component case is implicit. But it is proved that with appropriate operations and sequences employed, this problem can indeed be solved.

In this paper, we present a construction strategy of building rigid formation from non-rigid ones in two-dimensional space. The proposed strategy is developed based on three operations, namely, spindle splitting, rigid component shrink and edge floating. It is proved that the proposed strategy will guarantee the rigidity of obtained formation with minimum number of new links. We will also prove that the proposed construction sequence will cover all the possible cases.

\section{PReliminaries}

The structure maintenance of a multi-agent formation $F$ is achieved by preserving certain distances between designated pairs of agents. And a undirected graph $G=\{V, E\}$ can be employed to represent the composition of $F$, where $V$ and $E$ denote the vertex and edge set of $G$ respectively. Each agent within $F$ is represented by a vertex from $V$, while each distance constraint between a pair of agents of $F$ will be depicted as an edge from $E$.

The operations and construction strategy proposed in the following will focus on the underlying graph of the multiagent formation, and the inserted edges mentioned stands for the behavior of building new links between corresponding pairs of agents within formation.

\section{A. Basic notions}

For an undirected graph $G$, a walk of length $r$ from vertex $i$ to $j$ is a sequence of $r+1$ adjacent vertices from $i$ to $j$. If $i=j$, with no other vertex appears more than once, this walk is called a cycle. If there's a walk between any two vertices, $G$ is then mentioned as connected, and $G$ is called a tree if $G$ contains no cycles. If there are $m$ vertices in the neighbor set of $i$, then vertex $i$ is mentioned as a vertex with degree- $m$. A circle graph $G=\{V, E\}$ satisfy that $|V| \geq 4$ and $|V|=|E|$, while all of the vertices are of degree-2. A chain is obtained by removing one edge from a circle.

For vertex $i, N_{i}$ denotes its neighbor set, and $e_{i j}$ denotes the edge connecting $i$ and $j . E^{L}$ stands for the minimum inserted edge set in the process of construction.

\section{B. Graph rigidity}

Theorem 2.1: (Laman [8]) A graph $G=\{V, E\}$ is rigid in two-dimensional space if and only if there exists a subgraph $G^{\prime}=\left\{V, E^{\prime} \subset E\right\}$ of $G$ such that $\left|E^{\prime}\right|=2|V|-3$, and for any non-void vertex set $V^{\prime} \subset V$ with edge set $E^{\prime \prime} \subset E$ incident to $V^{\prime}$, there will be $\left|E^{\prime \prime}\right| \leq 2\left|V^{\prime}\right|-3$.

An arbitrary formation $F$ is mentioned as rigid if the underlying graph $G=\{V, E\}$ is rigid.

Definition 2.1: For a non-rigid graph $G^{\prime}\left\{V^{\prime}, E^{\prime}\right\}$, a rigid component is a maximal rigid subgraph of $G^{\prime}$ [1].

\section{Problem statement}

The main goal of this paper is to find strategies for obtaining rigid formation by connecting non-rigid components. The objectives of pursued strategies are listed as follow:

1. The proposed construction sequence will preserve the initial topology of non-rigid components, which means the process contains no removal of edges.

2. The construction sequence will guarantee the rigidity of obtained formation.

3. Minimum number of new links will be inserted during the construction process.

Throughout this paper, the only requirement for non-rigid component formation $F^{\prime}$ is that the underlying graph $G^{\prime}$ is connected. Even if $G^{\prime}$ is not connected, that is $F^{\prime}$ contains several separated sub-formations, the proposed construction sequence is still applicable. Thus here we assume that $G^{\prime}$ is connected only for simplicity of statement.

For two arbitrary non-rigid formations $F^{\prime}$ and $F^{\prime \prime}$, the problem of constructing rigid formation from this two nonrigid components can be formulated as finding the minimum inserted edge set between their underlying graph $G^{\prime}$ and $G^{\prime \prime}$ :

Problem 2.1: Given two arbitrary connected but non-rigid graphs $G^{\prime}$ and $G^{\prime \prime}$, design a strategy of discovering the minimum inserted edge set $E^{L}$, where $\forall e_{k m} \in E^{L}$, there will be $k \in G^{\prime}, m \in G^{\prime \prime}$, and the obtained graph $\left\{G^{\prime} \cup G^{\prime \prime} \cup\right.$ $\left.E^{L}\right\}$ is rigid, while $\left\{G^{\prime} \cup G^{\prime \prime} \cup E^{L} \backslash e_{k m}\right\}$ is not.

\section{OPERATIONS OF RIGID FORMATION CONSTRUCTION}

To recover the rigidity of graph from non-rigid components, one need to choose a correct collection of vertices on which the inserted edges will be attached. To begin with, a special kind of vertex need to be defined.

For a vertex $i$ within a undirected graph $G\{V, E\}$, if one of the following conditions holds for this $G$ and $i$, then $i$ will be mentioned as a spindle agent, as shown in Fig.2:

1: Vertex $i$ is from a rigid component $G_{r}\left\{V_{r}, E_{r}\right\}$ of $G$, and there exists a vertex $j \in N_{i}$ that satisfies $e_{i j} \notin$ $E_{r}$ and $e_{i j} \in E$.

2: There is no rigid component contained in $G$, for a cycle $C_{n}(n>3)$ within $G$ satisfying $\forall j, k \in C_{n}, e_{j k} \notin E$, vertex $i \in C_{n}$ satisfies that $\exists l \in N_{i}, e_{i l} \notin C_{n}$ and $e_{i l} \in E$.

3: $G$ is a tree graph, and there is a vertex $i \in G$ that satisfies $\left|N_{i}\right|>2$.

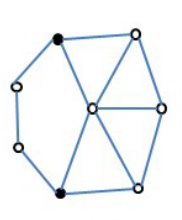

(a)

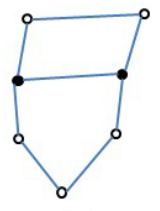

(b)

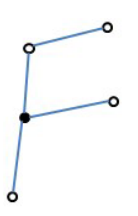

(c)
Fig. 2. The dotted vertices within the three graphs are the examples of spindle agents corresponding to condition 1, 2 and 3 respectively.

Actually the existence of spindle agent is the indicator of non-rigidity, one can easily figure out that for an arbitrary connected by non-rigid graph, there will be at least one spindle agent contained. 
Here we propose our first operation that will be employed in the merging sequence:

Definition 3.1: Consider a non-rigid graph $G^{\prime}$ containing some spindle agents $i, j, k, l$, the operation spindle splitting denotes that by dividing these vertices into several pieces, $G^{\prime}$ will be separated into $m$ unconnected subgraphs $G_{1}^{\prime} \ldots G_{m}^{\prime}$, where each of the subgraphs contains one piece of spindle agents generated from the splitting, while no spindle agents are contained in any of the obtained subgraphs, see Fig. 3.

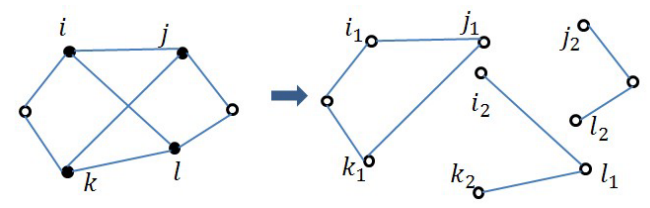

Fig. 3. Spindle splitting within non-rigid graph, the spindle agents $i, j, k, l$ are spilt such that the obtained subgraphs contains no spindle agents

Proposition 3.1: The operation of spindle splitting can always separate an arbitrary non-rigid graph into subgraphs containing only the three basic graphs of chain, circle and rigid components.

Proof: For an arbitrary non-rigid graph $G^{\prime}$, if $G^{\prime}$ contains no cycles, then $G^{\prime}$ will be a tree graph, and it is obviously all the subgraphs will be chain after spindle splitting. If $G^{\prime}$ contains cycles, start a walk from some vertex $m$ of degree-1, until the first vertex $k$ which is within a cycle, then this vertex $k$ must be a spindle agent. By spindle splitting a chain graph will be obtained from vertex $m$ to $k_{1}$.

If the non-rigid graph $G^{\prime}$ contains no vertices of degree1 , by definition all the spindle agents will hide among the shared edges between rigid components and circles. One can firstly figure out all the spindle agents contained in rigid components, and spilt these agents to obtain rigid components. The next step is to identify spindle agents within circles, and the splitting operation will leads to either circles or chains. If no rigid components are contained in $G^{\prime}$, then the splitting operation can start from a circle.

Note that the topology of obtained subgraphs are not unique, which are determined by the sequence of spindle splitting operations.

Proposition 3.2: To obtain a minimally rigid graph by adding a single agent $l$ to a chain graph consisting of $m$ vertex, $m$ edges need to be inserted, while when adding to a circle containing $m$ vertexes with $l, m-1$ edges is required.

Proof: To obtain a rigid graph from a chain graph and a single agent $l$, if $m$ edges are inserted, then there will be one edge attached on each of the vertices. Without loss of generality, denote one of the degree- 1 vertex of chain $v_{1}$, then by inserting one extra edge to its neighbor $v_{2}$, the graph $\left\{l \cup v_{1} \cup v_{2}\right\}$ is minimally rigid. Sequentially all the neighbors of $v_{2}$ can be considered as vertex addition operations by inserting edges between $v_{i-1}$ and $l$, thus the obtained graph will be minimally rigid. Similarly we can prove that with $m-1$ edges inserted between circle and single agent $l$, the new graph is minimally rigid.

If more than one circles are included in a non-rigid graph, the solutions of $E^{L}$ will be complicated comparing with Proposition 3.2. In order to reduce the complexity of construction, we present the second operation:

Definition 3.2: For a non-rigid graph $G^{\prime}$ containing a rigid component $G^{*}$, the term shrink denotes the operation of replacing $G^{*}$ with vertex pair $G_{r}\left(V_{r}, E_{r}\right)$, where $V_{r}=$ $\{i \cup j\}, i, j \in G^{*}, E_{r}=\left\{e_{i j}\right\}$, as shown in Fig.4.

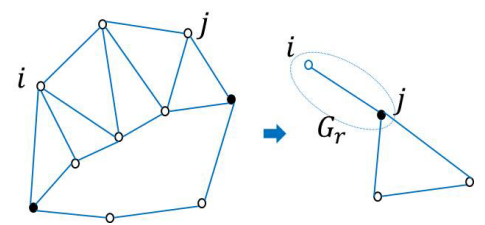

Fig. 4. Rigid component shrink will merge spindle agents together.

It is clear if a circle shares some edges with a rigid component, there will be at most two spindle agents existing at the same time, thus the operation of rigid component shrink can always be applied when there are no more than two spindle agents contained. In the next session we will show that in the reconstruction process, the operation of rigid component shrink can always be applied.

Lemma 3.1: To generate a rigid graph from a non-rigid graph $G^{\prime}$ and a single agent $l$, if $G^{\prime}$ contains rigid component $G^{*}$, shrink $G^{*}$ to obtain $G_{s}^{\prime}=\left\{G^{\prime} \backslash G^{*} \cup G_{r}\right\}$. Then for an inserted edge set $E^{L}$, if $\left\{G_{s}^{\prime} \cup E^{L} \cup l\right\}$ is minimally rigid then $\left\{G^{\prime} \cup E^{L} \cup l\right\}$ is rigid or minimally rigid, as shown in Fig.5.

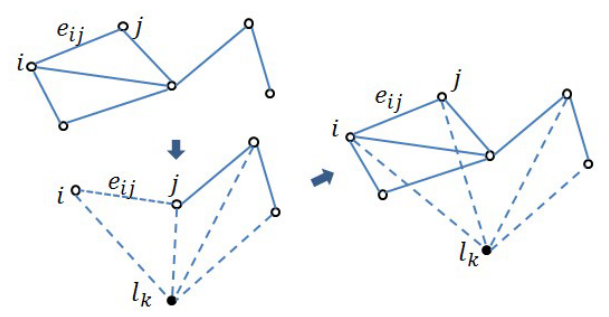

Fig. 5. Rigid component shrink in non-rigid graph will not change the requirement of inserted edge set $E^{L}$.

Proof: Referring to the basic operation of vertex addition of Henneberg sequence, at least two vertices from $G^{*}$ should be connected with $l$ by inserted edges in order to obtain a rigid graph from $G^{*}$ and $l$. Thus the operation of rigid component shrink is equivalent to choosing two vertices from $G^{*}$ such that the single agent $l$ can be connected to $G^{*}$. The process of inserting $l$ with other agents can be viewed as sequential vertex addition operations thus the obtained graph $\left\{G_{s} \cup E^{L} \cup l\right\}$ is minimally rigid. Specially, if $G^{*}$ is redundantly rigid, then $\left\{G^{\prime} \cup E^{L} \cup l\right\}$ will only be rigid.

This operation may seem to be trivial, but will greatly simplify the topology of non-rigid graph discussed in the later session. In fact this is the basis of the construction sequence mentioned in the next session.

It is known that vertex addition operation in Henneberg sequence to a rigid graph will preserve the rigidity. Similarly, the operation of edge floating can be developed: 
Definition 3.3: For a rigid graph, edge floating denotes the operation of removing one edge $e_{i k}$ for some vertex $i$ of degree 2 , and adding a new edge $e_{i l}$, where $k, l \in V$.

This operation can be intuitively depicted as one of the edge of vertex $i$ is floating over the rigid graph, of which an example can be found in Fig.6.

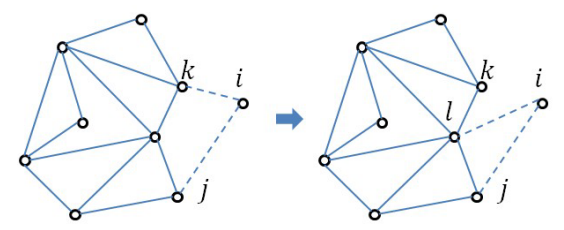

Fig. 6. Edge floating operation when adding a vertex to a rigid graph.

\section{CONSTRUCTION SEQUENCE OF RIGID FORMATION FROM NON-RIGID COMPONENTS}

There are two causes that will deprive a rigid graph of its rigidity, that is, breaking of edges, or loss of agents. In the rest of this section, we will deal with arbitrary nonrigid graphs distributed in two-dimensional space, without clarifying the cause of the non-rigidity.

After the introduction of spindle splitting and rigid component shrink in previous session, as well as the technics of obtaining a rigid graph from a basic non-rigid component and a single agent, we now present our construction sequence. The construction sequence will be presented step by step, starting from constructing a rigid graph from an arbitrary non-rigid graph and a single agent.

A. generating rigid graph from non-rigid component and single agent

This is the simplest condition of rigid graph construction with non-rigid components, which can be applied into the scenario of controlling a non-rigid formation of autonomous agents with a leader. The inserted edges can be understood as how to pick up some agents within formation to establish interaction links with the leader.

With Lemma 3.1 and Definition 3.1, we present the following construction sequence to obtain a rigid graph from non-rigid graph $G^{\prime}$ and a single agent $l$ :

Construction step $i$ : For an arbitrary non-rigid graph $G^{\prime}=$ $\left\{V^{\prime}, E^{\prime}\right\}$, perform the operation of spindle splitting to each of the spindle agents contained in $G^{\prime}$, until $G^{\prime}$ is separated into isolated subgraphs $G_{1}^{\prime} \ldots G_{n}^{\prime}$, and any of these subgraphs are one of the three basic graphs, as shown in Fig.7(a).

Construction step ii: Since $G^{\prime}$ contains cycles, then there will be at least one circle in the $n$ separated subgraphs, without loss of generality, let $G_{1}^{\prime}$ be a circle. Apply Proposition 3.2 such that $G_{1}^{L}=\left\{V_{1}^{\prime} \cup l, E_{1}^{\prime} \cup E_{1}^{L}\right\}$ is rigid, see Fig.7(b).

Construction step iii: Test the rigidity of restored graph $\left\{G_{1}^{L} \cup G_{2}^{\prime}\right\}$. If it is not rigid, shrink $G_{1}^{L}$ into vertex pair $G_{r 1}$ containing $l$, where $l$ is not a spindle agent in the new subgraph $\left\{G_{r 1} \cup G_{2}^{\prime}\right\}$, then apply Proposition 3.2 to obtain $E_{2}^{L}$, see Fig.7(c).

Construction step iv: Repeat step iii, until all the edge sets $E_{1}^{L} \ldots E_{n}^{L}$ are obtained, then the desired minimum inserted edge set $E^{L}$ will be $E^{L}=\left\{E_{1}^{L} \cup \ldots \cup E_{n}^{L}\right\}$, as shown in Fig.7(d).

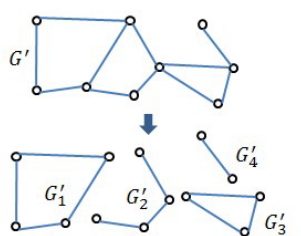

(a)

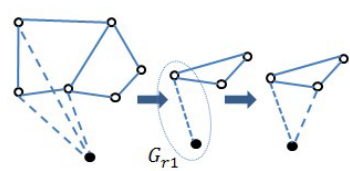

(c)

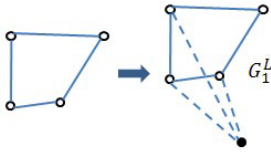

(b)

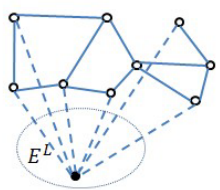

(d)
Fig. 7. Generating a rigid graph from non-rigid graph and single agent

For an arbitrary non-rigid graph, we have the following theorem:

Theorem 4.1: The proposed construction sequence will generate a minimally rigid graph from a non-rigid component and a single agent with minimum number of inserted edges.

Proof: Consider the operation in step ii. By Proposition 3.2 it is clear that the obtained graph is minimally rigid if $G_{1}^{\prime}$ is a circle, while the inserted set $E_{1}^{L}$ contains minimum number of edges. Then assume the obtained graph $G_{m-1}^{L}$ being minimally rigid and $E_{m-1}^{L}$ being a minimum edge set, we will prove that the obtained graph $G_{m}^{L}$ is minimally rigid, and $E_{m}^{L}$ is a minimum edge set as well.

Since $G_{m-1}^{L}$ is minimally rigid, it can then be regarded as a rigid component in restored subgraph $\left\{G_{m-1}^{L} \cup G_{m}^{\prime}\right\}$, then by the shrink operation, a vertex pair $\left\{l \cup v_{i}\right\}$ will be obtained, where $v_{i} \in G_{m-1}^{L}$. Then for $G_{m}^{\prime}, v_{i}$ will be the spindle agent connecting $G_{m}^{\prime}$ and $l$. Since a vertex pair is attached on $G_{m}^{\prime}$, it will be one of the three basic graphs thus Proposition 3.2 can be applied, which means that the obtained graph $G_{m}^{L}$ is minimally rigid, and $E_{m}^{L}$ is a minimum edge set.

Thus the final edge set $E^{L}=\left\{E_{1}^{L} \cup \ldots \cup E_{n}^{L}\right\}$ contains minimum number of edges and will guarantee the rigidity of obtained graph.

Remark 4.1: Note that in the shrink operation in step iii, the other vertex other than $l$ in each vertex pair $G_{r i}$ is chosen randomly from each rigid component $G_{i}^{L}$. Then by choosing different vertex pair in each shrink operation, Theorem 4.1 will cover all the possible solutions of merging process.

B. generating rigid graph from non-rigid component and rigid graph

Similar to the construction process with non-rigid graph and single agent, we start with a basic proposition:

Proposition 4.1: To obtain a rigid graph from a basic nonrigid graph, such as chain or circle graph $G^{\prime}=\left\{V^{\prime}, E^{\prime}\right\}$, and a minimally rigid graph $G=\{V, E\}$, the following approach can be applied:

1. Pick up a vertex $i$ from $G$, and apply Proposition 3.2 to obtain a rigid component $\bar{G}^{\prime}=\left\{V^{\prime} \cup i, E^{\prime} \cup E_{i}^{L}\right\}$. 
2. $\forall j \in V, \forall k \in V^{\prime}$, add another edge $e_{k j}$.

Then the obtained graph $G^{L}=\left\{G^{\prime} \cup G \cup E^{L}\right\}$ is minimally rigid, where $E^{L}=\left\{E_{i}^{L} \cup e_{k j}\right\}$ is the minimum inserted edge set, as shown in Fig.8.

Proof: Adding $i$ to $G^{\prime}$ is the same as vertex addition to a non-rigid graph thus $E_{i}^{L}$ is a minimum inserted edge set. In the second step, $i$ is a spindle agent. So if we take the shrink operation on $G^{\prime}$ and $G$, a three vertex chain graph $\{k, i\} \cup$ $\{i, j\}$ will be obtained, where $k$ and $j$ is randomly chosen from $V^{\prime}$ and $V$. According to Lemma 3.1, by inserting $e_{k j}$, the obtained graph $G^{L}=\left\{G^{\prime} \cup G \cup E^{L}\right\}$ will be minimally rigid, and $E^{L}$ will be a minimum edge set.

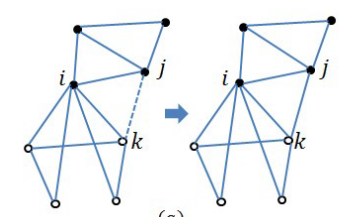

(a)

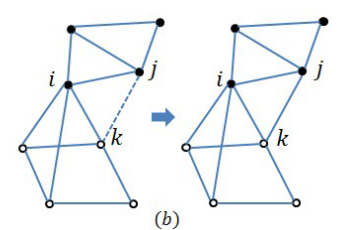

(b)

\section{$\square$}

Fig. 8. Generating rigid graph from basic graphs of chain and circle

Lemma 4.1: For a rigid graph generated by merging a circle or chain with rigid graph, if one performs the operation of edge floating on any of the inserted edge under the following conditions:

1. Not all the inserted edges start from or sink at a same vertex.

2. No reduplicative edges are introduced.

Then the obtained graph is still rigid.

Proof: By Proposition 4.1 the initial graph is rigid, and the operation of edge floating on any of the inserted edge does not change the total number of edges, as shown in Fig.9.

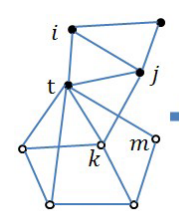

(a)

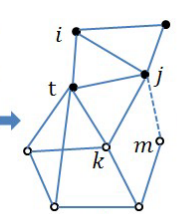

(b)

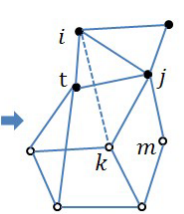

(c)
Fig. 9. Edge floating will not change the rigidity of given graph.

If we remove edge $e_{m t}$, then the obtained graph can be viewed as adding a single agent $m$ to a non-rigid graph $G_{m}$. By the aforementioned construction sequence of adding a single agent to non-rigid graph, the inserted edge $e_{m j}$ will recover the rigidity, where $j$ can be any vertex from rigid graph $G$. Specially, if $\left|E^{L}-1\right|$ edges start from or sink at the same vertex $p, p$ will become the spindle agent when inserting the next edge, so it is clear that $e_{m p}$ can not recover the rigidity, which means the inserted edge can not start from or ends at a same vertex.

Remark 4.2: Lemma 4.1 covers all the possible solution set $E^{L}$ when constructing a rigid graph from a basic nonrigid graphs and a rigid graph.

With Proposition 4.1 and lemma 4.1, we present the following sequence of merging non-rigid graph $G^{\prime}=\left\{V^{\prime}, E^{\prime}\right\}$ with rigid graph $G=\{V, E\}$ :
Construction step $i$ : For an arbitrary non-rigid graph $G^{\prime}\left\{V^{\prime}, E^{\prime}\right\}$, perform the operation of spindle splitting to each of the spindle agents contained in $G^{\prime}$, thus $G^{\prime}$ is separated into isolated subgraphs $G_{1}^{\prime} \ldots G_{n}^{\prime}$, and any of these subgraphs are one of the three basic graphs, see Fig.10(a).

Construction step ii: Since $G^{\prime}$ contains cycles, then there will be at least one circle in the $n$ separated subgraphs. Without loss of generality, let $G_{1}^{\prime}$ be a circle. Apply Proposition 4.1 to $G_{1}^{\prime}$ and $G$ such that $G_{1}^{L}=\left\{V_{1}^{\prime} \cup G, E_{1}^{\prime} \cup E_{1}^{L}\right\}$ is minimally rigid, see Fig.10(b).

Construction step iii: Test the rigidity of restored graph $\left\{G_{1}^{L} \cup G_{2}^{\prime}\right\}$. If it is not rigid, shrink $G_{1}^{L}$ into agent pair $G_{r 1}$ containing any one vertex from $V$, then for the new subgraph $\left\{G_{r 1} \cup G_{2}^{\prime}\right\}$, apply Proposition 4.1 to obtain $G_{2}^{L}$ and $E_{2}^{L}$, see Fig.10(c).

Construction step iv: Repeat step iii, until all the edge sets $E_{1}^{L} \ldots E_{n}^{L}$ are obtained, then the desired minimum inserted edge set $E^{L}$ will be $E^{L}=\left\{E_{1}^{L} \cup \ldots \cup E_{n}^{L}\right\}$, as shown in Fig.10(d).

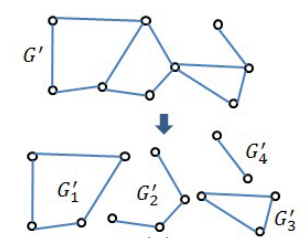

(a)

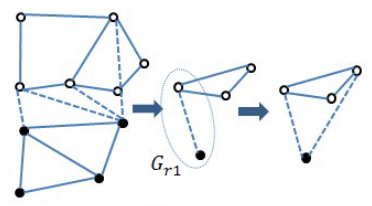

(c)

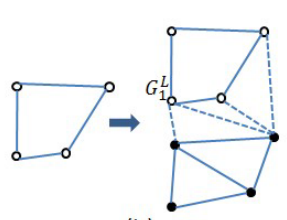

(b)

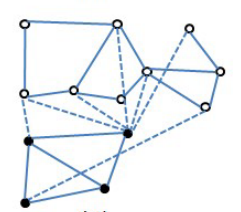

(d)
Fig. 10. Generating a rigid graph from non-rigid and rigid component.

Theorem 4.2: The proposed construction sequence will generate a minimally rigid graph from a non-rigid component and a rigid graph with minimum number of inserted edges.

The proof is similar to Theorem 4.1 and is omitted.

Remark 4.3: Theorem 4.2 covers all the possible solution of merging non-rigid and rigid graph, if Lemma 4.1 is applied in the step $i i$ and $i i i$ of the proposed construction process.

\section{C. generating rigid graph from non-rigid components}

Here the construction sequence of two arbitrary non-rigid graphs is proposed as our main result, starting with a basic proposition:

Proposition 4.2: To obtain a rigid graph from two chain or circle graphs $G_{1}^{\prime}$ and $G_{2}^{\prime}$, the following approach can be applied,:

1. Apply Proposition 3.2 to $G_{1}^{\prime}$ and $i \in G_{2}^{\prime}$ to obtain a inserted edge set $E_{i}^{L}$. Then $\forall k \in G_{1}^{\prime}$ and $\forall j \in N_{i}$, insert another edge $e_{k j}$, such that the obtained graph $G_{1}^{L}=\left\{G_{1}^{\prime} \cup\right.$ $\left.i \cup j \cup E_{i}^{L} \cup e_{k j}\right\}$ is minimally rigid.

2. Shrink $G_{1}^{L}$ into vertex pair containing one agent from $G_{1}^{\prime}$ and apply Proposition 3.2 again.

Then the obtained graph is minimally rigid, see Fig.11. 
Proof: The first part is a vertex addition operation to a non-rigid graph, while the insertion of edge $e_{k j}$ can be viewed as a vertex addition operation of vertex $j$.

The second part can be viewed as generating a rigid graph from a basic non-rigid graph and a rigid graph, thus the proof is clear with reference to the proof of Proposition 4.1.

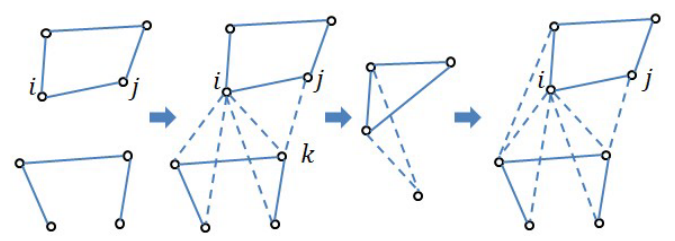

Fig. 11. Generating a rigid graph from two basic non-rigid components

Similar to Lemma 4.1, we have the following result:

Lemma 4.2: Take the operation of edge floating to the graph obtained by Proposition 4.2, under the following conditions:

1. Not all the inserted edges start from or sink at a same vertex.

2. No reduplicative edges are introduced.

Then the obtained graph is still rigid.

The proof is similar to Lemma 4.1 and is omitted.

With Proposition 4.2 and lemma 4.2, we present the sequence of non-rigid graph merging.

Construction step $i$ : For two arbitrary non-rigid graph $G^{\prime}$ and $G^{\prime \prime}$ in Fig.12(a), perform the operation of spindle splitting to each of the spindle agents contained in this two graph, such that $G^{\prime}$ and $G^{\prime \prime}$ are separated into isolated subgraphs $G_{1}^{\prime} \ldots G_{n}^{\prime}, G_{1}^{\prime \prime} \ldots G_{m}^{\prime \prime}$, and any of these subgraphs are one of the three basic graphs, as shown in Fig.12(b).

Construction step ii: Since $G^{\prime}$ and $G^{\prime \prime}$ both contains cycles, let $G_{1}^{\prime}$ be a circle. Apply Proposition 4.2 to obtain a rigid subgraph $G_{11}^{L}$ from $G_{1}^{\prime}$ and $G_{1}^{\prime \prime}$, then apply Proposition 4.1 to $G_{11}^{L}$ and the rest of subgraphs generated from $G^{\prime \prime}$ until all these subgraphs are neutralized to obtain a minimally rigid graph $G_{1}^{L}$, see Fig.12(c).

Construction step iii: Test the rigidity of restored graph $\left\{G_{1}^{L} \cup G_{2}^{\prime}\right\}$. If it is not rigid, shrink $G_{1}^{L}$ into agent pair $G_{r 1}$ containing one vertex from $V_{2}^{\prime}$, then for the new subgraph $G_{r 1} \cup G_{2}^{\prime}$, apply Proposition 4.1 to obtain a minimally rigid graph, repeat this step again until all the separated subgraphs generated from $G^{\prime}$ are neutralized, see Fig.12(d).

Theorem 4.3: The proposed construction sequence will generate a minimally rigid graph from two non-rigid components with minimum number of inserted edges.

This process can be viewed as a sequential merging of a rigid graph with non-rigid ones, thus the proof is omitted.

Remark 4.4: Theorem 4.3 covers all the possible construction solutions with two non-rigid graph, if Lemma 4.1 and 4.2 are applied in the construction process.

Corollary 4.1: If redundantly rigid components are contained in the initial non-rigid graph $G^{\prime}$, then the obtained graph will only be rigid.

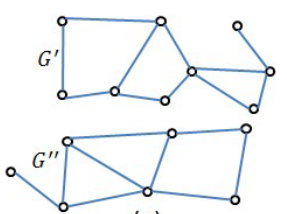

(a)

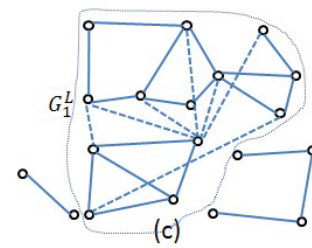

Fig. 12. Generating a rigid graph from two arbitrary non-rigid graphs

\section{Applications AND CONCLUSION}

In this paper, the construction of rigid formation with nonrigid components are discussed in two dimensional space, with three new operations introduced to guarantee the rigidity of obtained formation with minimum number of inserted links. Different scenarios of rigid formation constructions can be applied to recovering a larger rigid formation from nonrigid formations for sophisticated tasks. These approaches can be realized in multi-agent cooperation tasks as a backup solution in the case of agent loss and interaction link broken.

Currently this paper focuses on the rigid formation construction in two-dimensional space. The future work may include the construction operations with multi non-rigid components, in both two and three dimensions.

\section{REFERENCES}

[1] A. R. Berg, and T. Jordan. "Algorithms for graph rigidity and scene analysis." Algorithms-ESA 2003. Springer Berlin Heidelberg, 2003. 78-89.

[2] T. H. Summers, C. Yu, and B. D. O. Anderson. "Addressing agent loss in vehicle formations and sensor networks." International Journal of Robust and Nonlinear Control, 2009, 19(15): 1673-1696.

[3] B. Fidan, J. M. Hendrickx, and B. D. O. Anderson. "Closing ranks in rigid multi-agent formations using edge contraction." International Journal of Robust and Nonlinear Control, 2010, 20(18): 2077-2092.

[4] T. Eren, B. D. O. Anderson, and AS. Morse, "Operations on rigid formations of autonomous agents", Communications in Information \& Systems, 2003, 3(4): 223-258.

[5] B. D. O. Anderson, C. Yu, and B. Fidan, "Rigid graph control architectures for autonomous formations." Control Systems, IEEE, 2008, 28(6): 48-63.

[6] T. Eren, B. D. O. Anderson, and AS. Morse, "Information structures to control formation splitting and merging." American Control Conference, 2004. Proceedings of the 2004. Vol. 6. IEEE, 2004.

[7] C. Yu, B. Fidan, B. D. O. Anderson, "Principles to control autonomous formation merging." American Control Conference, 2006. IEEE, 2006.

[8] G. Laman, "On graphs and rigidity of plane skeletal structures." Journal of Engineering mathematics, 1970, 4(4): 331-340. 